\title{
Enantioselective room temperature phosphorescence detection of non-phosphorescent analytes based on interaction with $\beta$-cyclodextrin/1-bromonaphthalene complexes
}

\author{
Carmen García-Ruiz ${ }^{1}$, XueShan $\mathrm{Hu}^{2}$, Freek Ariese, Cees Gooijer* \\ Department of Analytical Chemistry and Applied Spectroscopy, Laser Centre, Vrije Universiteit, \\ De Boelelaan 1083, NL-1081 HV Amsterdam, The Netherlands
}

Received 3 June 2004; received in revised form 30 September 2004; accepted 1 December 2004

Available online 7 January 2005

\begin{abstract}
Menthol (MT) induces strong room temperature phosphorescence (RTP) of 1-bromonaphthalene (1BrN) in aqueous $\beta$-cyclodextrin ( $\beta$-CD) suspensions, even under non-deoxygenated conditions. Interestingly, (-)-MT and (+)-MT enantiomers give rise to different phosphorescence intensities, the difference being $19 \pm 3 \%$. It is argued that the signal can be mainly ascribed to the formation of ternary complexes $\beta$ CD/1BrN/MT which show different RTP lifetimes, i.e. $4.28 \pm 0.06$ and 3.71 $\pm 0.06 \mathrm{~ms}$ for (-)-MT and (+)-MT, respectively. Most probably, the stereochemical structure of (-)-MT provides a better protection of $1 \mathrm{BrN}$ against quenching by oxygen than $(+)$-MT. This interpretation is in line with the observation that under deoxygenated conditions the phosphorescence intensity difference for the two complexes becomes very small, i.e. only about $4 \%$.

The lifetime difference under aerated conditions enables the direct determination of the MT stereochemistry. For mixtures, in view of the $0.06 \mathrm{~ms}$ uncertainty in the lifetime, enantiomeric purity can be determined down to $10 \%$. Furthermore, in the case of MT the concentration of the least abundant enantiomer should be at least $3 \times 10^{-4} \mathrm{M}$, since otherwise complex dissociation would obscure the lifetime difference. (c) 2004 Elsevier B.V. All rights reserved.
\end{abstract}

Keywords: Menthol; Ternary complex; Chiral discrimination; Phosphorescence lifetime

\section{Introduction}

The observation of room temperature phosphorescence (RTP) in liquid solutions or suspensions generally requires thorough deoxygenation. Interestingly, there are exceptions to this rule: intense RTP of 1-bromonaphthalene $(1 \mathrm{BrN})$ in aqueous $\beta$-cyclodextrin $(\beta-C D)$ solutions has been observed under aerated conditions in the presence of alcohols (tert-butyl alcohol, cyclohexanol, cyclopentanol, and 1-pentanol) [1-3], alkanes (cyclohexane, bromocyclohex-

\footnotetext{
* Corresponding author. Tel.: +3120 4447524; fax: +31 204447543 .

E-mail address: gooijer@few.vu.nl (C. Gooijer).

${ }^{1}$ Present address: Dept. of Analytical Chemistry, University of Alcalá, Spain.

2 Present address: Beijing Micro-Tech Institute, China.
}

ane, and 1,2-dibromoethane) [3], surfactants (triton X-100, sodium dodecyl sulfate, dodecylbenzene sulfonate, and deoxycholate) $[2,4,5]$, or polymers ( $p$-octylpolyethylene glycol phenylether) [6]. This phenomenon can be attributed to the formation of ternary complexes that protect the phosphorophore $(1 \mathrm{BrN})$ in the excited triplet $\left(\mathrm{T}_{1}\right)$ state from quenching by oxygen. Based on the formation of such ternary complexes, also various bromonaphtol derivatives have been detected by RTP under non-deoxygenated conditions [7,8]. Nazarov et al. have even reported strong signals for an organic phosphorophore not substituted with a heavy atom such as bromine: for naphthalene- $\beta-C D$-adamantane suspensions a phosphorescence lifetime as long as $10.3 \mathrm{~s}$ could be obtained [9].

Only very recently, some applications based on RTP in aerated aqueous suspensions have been developed. Zhai et 


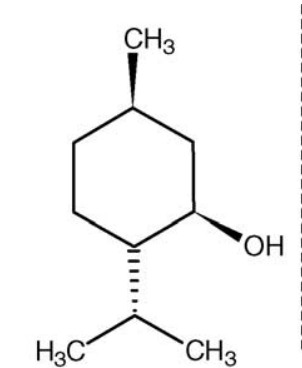

(1R,2S,5R)-(-)-Menthol

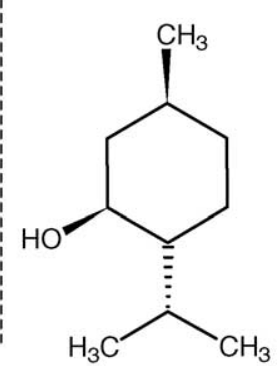

(1S,2R,5S)-(+)-Menthol
Fig. 1. Structure of the enantiomers of MT.

al. have demonstrated the use of 6-bromo-2-naphthol/ $\beta-C D$ as an RTP sensor for 28 small organic molecules, showing a high molecular recognition ability for cyclohexane [10]. Sanz-Medel and co-workers developed the first RTP sensor for $\mathrm{pH}$ based on the long-range Förster energy transfer from a phosphorophore (1BrN or 6-bromo-2-naphtyl sulfate, which emits RTP in aerated aqueous solutions) to a mixture of $\mathrm{pH}-$ sensitive dyes [11]. The Sanz-Medel group has also reported two different analytical methods for the determination of mercury [12] or cyanide [13] in water samples based on the RTP of $1 \mathrm{BrN} / \beta-\mathrm{CD}$.

Chiral compounds that show native phosphorescence can be distinguished by RTP if they form complexes with chiral ligands and if the resulting diastereoisomeric species have different spectroscopic properties. For instance, we have demonstrated the enantioselective detection of phosphorescent analytes (e.g., camphorquinone) in $\alpha-C D$ solutions, based on differences in phosphorescence lifetimes (see accompanying paper [14]). In the present paper we will consider the potential of RTP emitted by $1 \mathrm{BrN} / \beta-\mathrm{CD}$ complexes to distinguish between the enantiomers of a chiral, nonphosphorescent compound. We selected for this study the enantiomers of menthol (MT; see Fig. 1 for structures), a compound hardly detectable in the liquid phase by conventional spectroscopic means since it does not contain any chromophoric group.

\section{Experimental section}

\subsection{Chemicals and samples}

(1R,2S,5R)-(-)-MT, (1S,2R,5S)-(+)-MT, 1-bromonaphthalene $(1 \mathrm{BrN})$, and $\beta$-cyclodextrin $(\beta-\mathrm{CD})$ were purchased from Aldrich (Steinheim, Germany). Water used for the preparation of the solutions was purified through a Milli-Q system from Millipore (Bedford, MA, USA).

A stock solution with $1 \mathrm{BrN}$ and $\beta-\mathrm{CD}$ at nominal concentrations of $10^{-4}$ and $10^{-2} \mathrm{M}$, respectively, was prepared by weighing the appropriate amounts, adding Milli-Q water, and stirring for $1 \mathrm{~h}$. $\beta-\mathrm{CD}$ would be soluble at that level, but in the presence of $1 \mathrm{BrN}$ a cloudy suspension was obtained, in accordance with the literature $[12,13]$. However, no precipitation was observed, indicating that the total concentration (dissolved + suspended) was still equal to the nominal concentration.

Aqueous solutions of MT enantiomers $\left(10^{-3} \mathrm{M}\right)$ were prepared by weighing the appropriate amount of solid, adding Milli-Q water, and sonicating for $30 \mathrm{~min}$ until total dissolution.

Samples were prepared by mixing $1.5 \mathrm{~mL}$ of the $1 \mathrm{BrN} / \beta-$ CD suspension with $1.5 \mathrm{~mL}$ of an aqueous solution containing MT in the desired concentration or enantiomeric composition and vortexing for $30 \mathrm{~min}$. During this process the cloudy solution cleared, indicating sufficient solubility of the final mixture. In all experiments the final concentration of $1 \mathrm{BrN}$ was $5 \times 10^{-5} \mathrm{M}$ and that of $\beta-\mathrm{CD}$ was $5 \times 10^{-3} \mathrm{M}$; the concentration of MT was $5 \times 10^{-4} \mathrm{M}$ unless otherwise indicated. We found that after 2 weeks of storage in the refrigerator $\left(\sim 4{ }^{\circ} \mathrm{C}\right)$, phosphorescence lifetimes would increase, indicating gradual aggregation. In practice, suspensions were measured within 1-4 days of preparation, and the lifetimes were checked before each series of measurements.

\subsection{Instrumentation}

An LS-50B luminescence spectrometer (Perkin-Elmer, Beaconsfield, UK) equipped with a flash xenon arc source was used for phosphorescence measurements. Excitation and emission slits were $10 \mathrm{~nm}$; excitation and emission wavelengths were set at 285 and $528 \mathrm{~nm}$, respectively. In order to remove interfering fluorescence a delay time of $0.10 \mathrm{~ms}$ was used; the gating time was typically $5.00 \mathrm{~ms}$. For the lifetime measurements the gating time was set at $0.10 \mathrm{~ms}$, while the delay times were varied in steps of $0.10 \mathrm{~ms}(1 \mathrm{BrN} / \beta$ $\mathrm{CD}$ solutions) or in steps of $0.5 \mathrm{~ms}(1 \mathrm{BrN} / \beta-\mathrm{CD}$ solution in the presence of menthol enantiomers). Triplet lifetime values were obtained by fitting the RTP decay curves to a mono- or bi-exponential decay curve (Origin 6.1, OriginLab Corporation).

For comparison, phosphorescence spectra under deoxygenated conditions were recorded in a special long-neck luminescence cuvette (Hellma Benelux BV, The Netherlands) with a cap and Teflon tubes, so that a low nitrogen flow through the suspension could be maintained. Fluorescence measurements were performed using an excitation wavelength of $276 \mathrm{~nm}$, an emission wavelength at $336 \mathrm{~nm}$, and excitation and emission slits of $10 \mathrm{~nm}$.

\section{Results and discussion}

It should be noted that $1 \mathrm{BrN} / \beta-\mathrm{CD}$ complexes in aqueous systems are prone to aggregate formation, a phenomenon that obviously will also affect the ternary complex formation with menthol. Therefore, careful sample preparation procedures had to be developed in order to achieve reproducible results. It was observed that mixing of the solutions could 
not be performed by sonication since the accompanying temperature rise affected the results; vortexing turned out to be the mixing method of choice. We investigated the influence of the vortexing time on the phosphorescence intensity of $1 \mathrm{BrN} / \beta-\mathrm{CD}$ in the presence of menthol enantiomers. The intensity increased during the first $10 \mathrm{~min}$, then levelled off and a more or less stable signal was obtained for vortexing times up to $60 \mathrm{~min}$. For practical reasons, all suspensions were vortexed for $30 \mathrm{~min}$ before the spectra were recorded.

In line with the literature [1], upon addition of menthol we propose the formation of a ternary complex $1 \mathrm{BrN} / \beta-\mathrm{CD} / \mathrm{MT}$. Interestingly, Fig. 2 (full lines) shows that the phosphorescence intensities of the $1 \mathrm{BrN} / \beta-\mathrm{CD}$ suspension in the presence of the two MT enantiomers are different, the difference being $19 \pm 3 \%$. In contrast, the much weaker fluorescence spectra of the complex in the presence of the two MT enantiomers were of equal intensity (spectra not shown). These results can be understood if we assume that the complex structures of $1 \mathrm{BrN} / \beta-\mathrm{CD} / \mathrm{MT}$ are not identical for (-)- and (+)MT, and that these differences affect the phosphorescence yields, but not the fluorescence yields. Presumably there is a difference in protection against oxygen quenching. To test this hypothesis, lifetimes of the aqueous $1 \mathrm{BrN} / \beta-\mathrm{CD}$ suspension in absence and presence of menthol enantiomers were measured.

As shown in Fig. 2 (lower spectrum), also in absence of menthol does $1 \mathrm{BrN} / \beta-\mathrm{CD}$ show RTP in non-deoxygenated aqueous suspension, though of a relatively low intensity. This indicates that also for the binary complex there is already a substantial protection of the $\mathrm{T}_{1}$-state of $1 \mathrm{BrN}$, fully in line with the findings reported by Turro et al. in the early 1980's [15]. The associated phosphorescence decay curve is shown in Fig. 3. It clearly fits to a bi-exponential decay as demonstrated by the residuals depicted in the lower part of this figure, whereas a mono-exponential decay curve does not describe the results properly. The two lifetimes obtained (see Table 1) can be assigned to two complex configurations. We assume that the binary complex $1 \mathrm{BrN} / \beta-\mathrm{CD}$ (denoted as (1)) shows the shorter lifetime $\tau_{1}$ of $0.13 \pm 0.01 \mathrm{~ms}$. Presumably, in addition $1 \mathrm{BrN}$ complexes with two cyclodextrins, such as $\beta-\mathrm{CD} / 1 \mathrm{BrN} / \beta-\mathrm{CD}(\mathbf{2})$ are formed, in which the triplet state of $1 \mathrm{BrN}$ is somewhat better protected so that its lifetime $\tau_{2}$ is substantially higher, i.e. $0.83 \pm 0.06 \mathrm{~ms}$. The pre-exponential weight factors $A_{1}$ and $A_{2}$ in Table 1 point to a ratio of 2:1 for complexes (1) and (2).

Also for the longer-lived phosphorescence decay curves obtained in the presence of MT a bi-exponential fit was needed; a mono-exponential fit clearly did not result in randomly distributed residuals (see Fig. 4). Interestingly, the longer lifetimes were significantly different for the two enantiomers, i.e. $3.71 \pm 0.06$ and $4.28 \pm 0.06 \mathrm{~ms}$ for $(+)$-MT and (-)-MT, respectively. The shorter lifetimes were identical in both suspensions, i.e. $0.94 \pm 0.06$ and $0.95 \pm 0.06 \mathrm{~ms}$, very close to that of structure (2), measured in absence of MT (see Table 1). Although two exponentials are sufficient to explain the experimental data for the MT complexes, it should be noted that a minor contribution from a short-living component - expected if complex (1) is still present - cannot be fully excluded.

Of the species contributing to the phosphorescence signal (Fig. 2, solid lines), the dominating one is the most interesting since it shows $15 \%$ RTP lifetime differences for the (+)-MT and the (-)-MT complexes. We attribute these long-living signals to ternary complexes $\mathrm{MT} / 1 \mathrm{BrN} / \beta-\mathrm{CD}(\mathbf{3})$, where MT

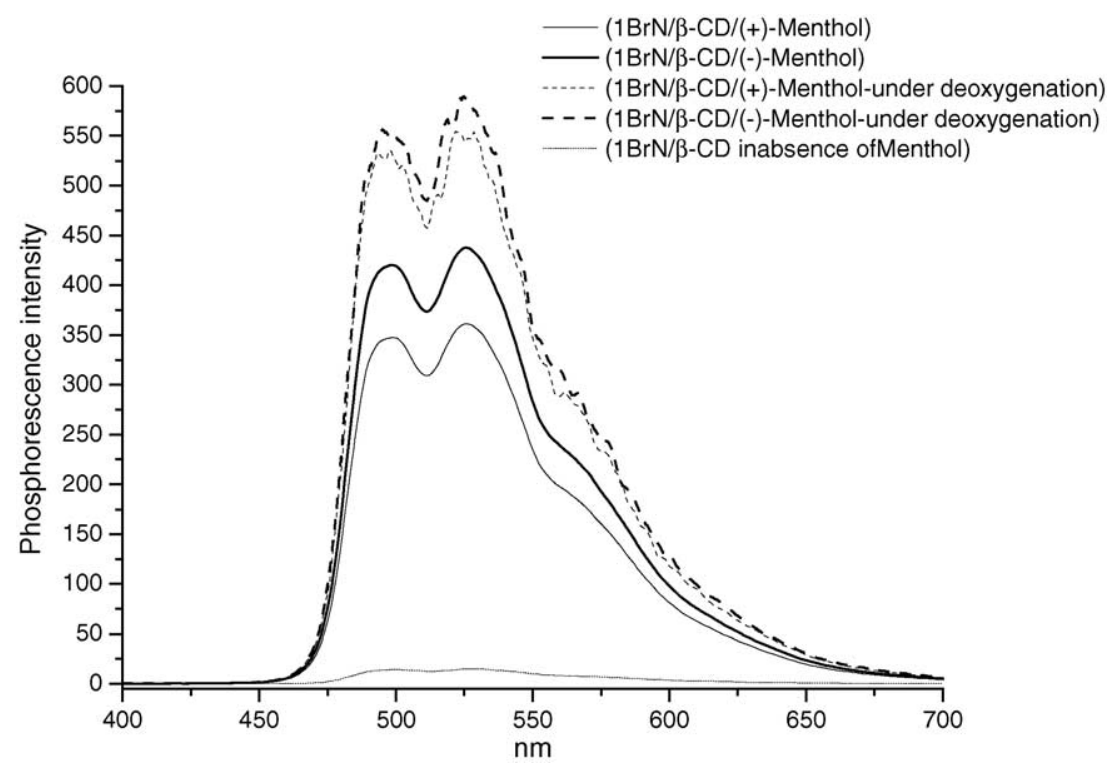

Fig. 2. Phosphorescence emission spectra of $1 \mathrm{BrN} / \beta-\mathrm{CD}$ suspension in presence of MT enantiomers, in air-equilibrated suspensions (full lines) and after deoxygenation (dashed lines). Concentrations $5 \times 10^{-3} \mathrm{M} \beta-\mathrm{CD}, 5 \times 10^{-5} \mathrm{M} 1 \mathrm{BrN}$ and $5 \times 10^{-4} \mathrm{M}$ of $(+)$ - or (-)-MT. The lower spectrum was recorded in absence of MT under aerated conditions. Spectrometer settings - delay time: $0.10 \mathrm{~ms}$, gating time: $5.00 \mathrm{~ms} ; \lambda_{\mathrm{exc}}=285 \mathrm{~nm}$, excitation and emission band widths: $10 \mathrm{~nm}$. 

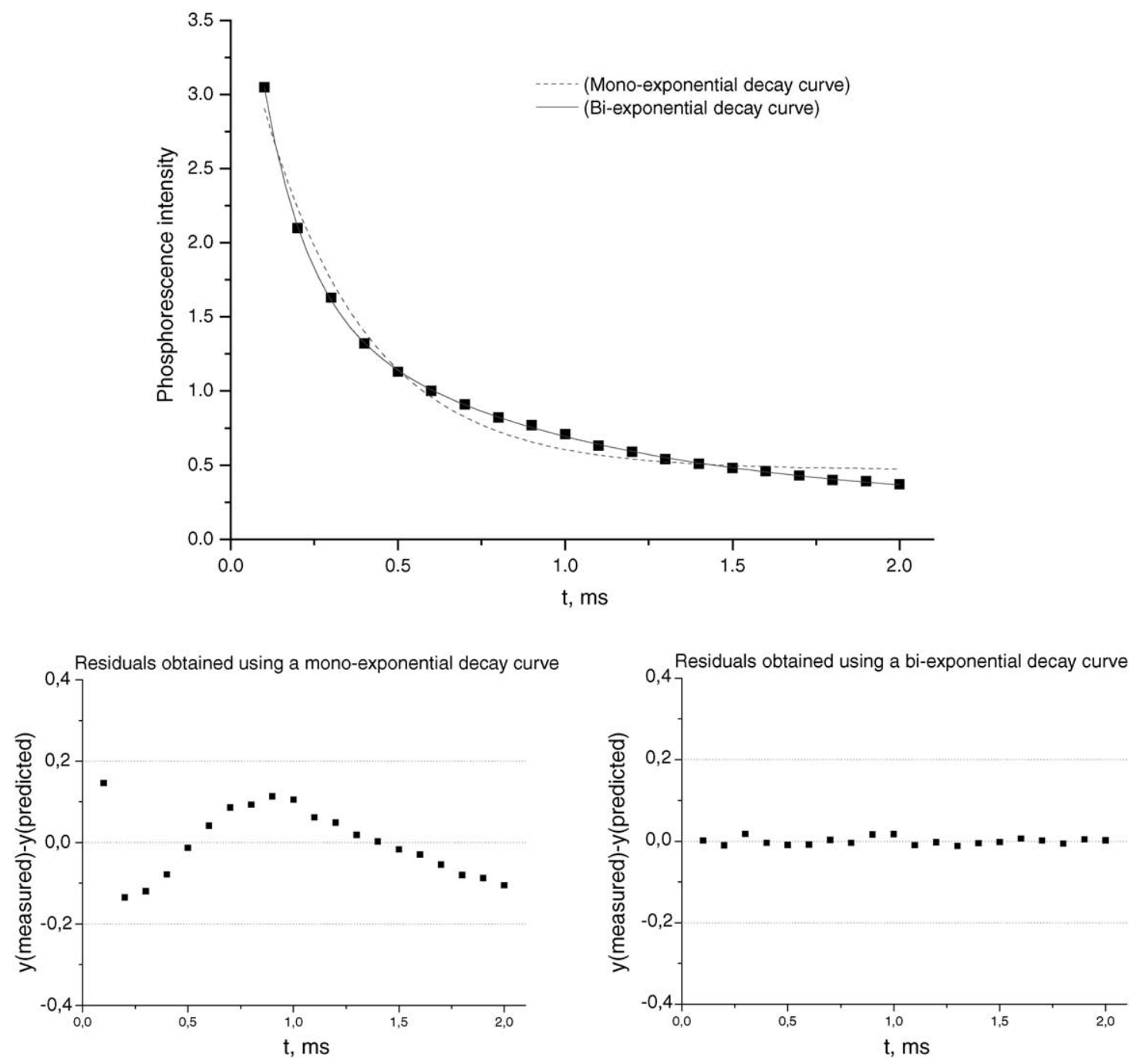

Fig. 3. Exponential decay curves and residual analysis for phosphorescence lifetimes of the $1 \mathrm{BrN} / \beta-\mathrm{CD}$ suspension under non-deoxygenated conditions.

provides protection of the $1 \mathrm{BrN}$ excited triplet state against oxygen quenching. Most probably, the stereochemical structure of (-)-MT provides a better protection after complexation with the chiral $\beta-\mathrm{CD}$ than in the case of (+)-MT.

If this interpretation is correct, it would be expected that under deoxygenated conditions, where the bimolecular quenching action of oxygen is insignificant, the RTP intensities of both enantiomeric complexes would become identical. As shown in Fig. 2 (upper curves) this is almost true: the remaining difference in intensities is only about $4 \%$. This may point to minor differences in complex formation constants (1) $\rightleftarrows(3)$ for (+)-MT and (-)-MT, in agreement with the different pre-exponential factors $A_{3}$ in Table 1 and with the different complex formation curves as will be shown below.

From the relative increases in phosphorescence in Fig. 2 upon deoxygenation we can estimate the lifetime $\tau_{0}$ in absence of quencher and the oxygen quenching rate constants. It should be realized that the extra phosphorescence is mainly due to an increased contribution from complex (2), since its phosphorescence lifetime will be more strongly enhanced

Table 1

Phosphorescence lifetimes $\left(\tau_{i}\right)$ and weight factors $\left(A_{i}\right)$ of the $1 \mathrm{BrN} / \beta-\mathrm{CD}$ suspension in absence and presence of MT enantiomers, obtained by fitting the experimental data to a bi-exponential decay curve: $y=A_{1} \exp \left(-x / \tau_{1}\right)+A_{2} \exp \left(-x / \tau_{2}\right)$

\begin{tabular}{|c|c|c|c|c|c|c|}
\hline \multirow[t]{2}{*}{ System } & \multicolumn{2}{|c|}{ (1) $1 \mathrm{BrN} / \beta-\mathrm{CD}$} & \multicolumn{2}{|c|}{ (2) $\beta-\mathrm{CD} / 1 \mathrm{BrN} / \beta-\mathrm{CD}$} & \multicolumn{2}{|c|}{ (3) $\mathrm{MT} / 1 \mathrm{BrN} / \beta-\mathrm{CD}$} \\
\hline & $A_{1}$ & $\tau_{1}(\mathrm{~ms})$ & $A_{2}$ & $\tau_{2}(\mathrm{~ms})$ & $\overline{A_{3}}$ & $\tau_{3}(\mathrm{~ms})$ \\
\hline $\begin{array}{l}1 \mathrm{BrN} / \beta-\mathrm{CD}\left(R^{2}=0.9998\right) \\
(+)-\mathrm{MT} / 1 \mathrm{BrN} / \beta-\mathrm{CD}\left(R^{2}=0.9998\right) \\
(-)-\mathrm{MT} / 1 \mathrm{BrN} / \beta-\mathrm{CD}\left(R^{2}=0.9998\right)\end{array}$ & $3.15 \pm 0.06$ & $0.13 \pm 0.01$ & $\begin{array}{l}1.54 \pm 0.05 \\
5.87 \pm 0.31 \\
5.43 \pm 0.27\end{array}$ & $\begin{array}{l}0.83 \pm 0.06 \\
0.94 \pm 0.06 \\
0.95 \pm 0.06\end{array}$ & $\begin{array}{l}11.92 \pm 0.33 \\
13.84 \pm 0.28\end{array}$ & $\begin{array}{l}3.71 \pm 0.06 \\
4.28 \pm 0.06\end{array}$ \\
\hline
\end{tabular}




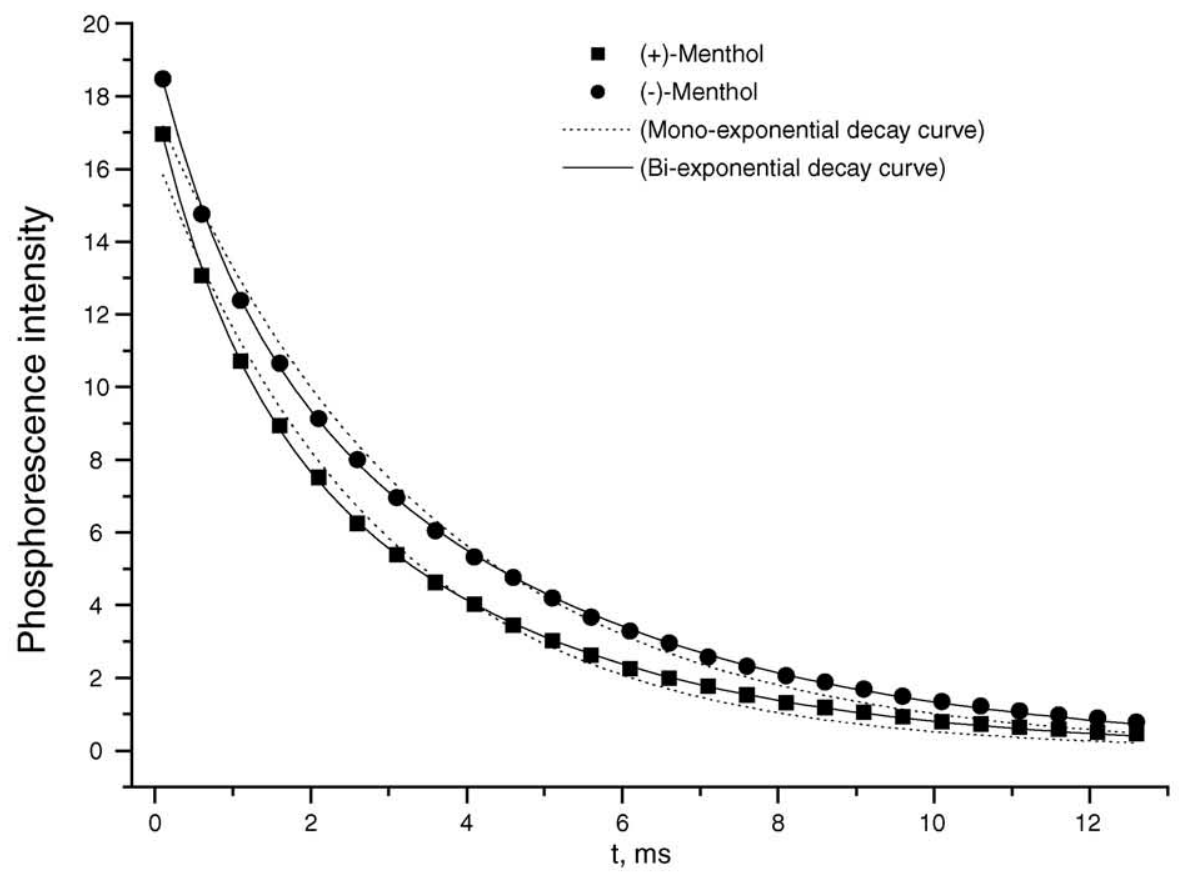

$1 \mathrm{BrN} / \beta-\mathrm{CD} /(+)-$ Menthol

$1 \mathrm{BrN} / \beta-\mathrm{CD} /(-)-$ Menthol
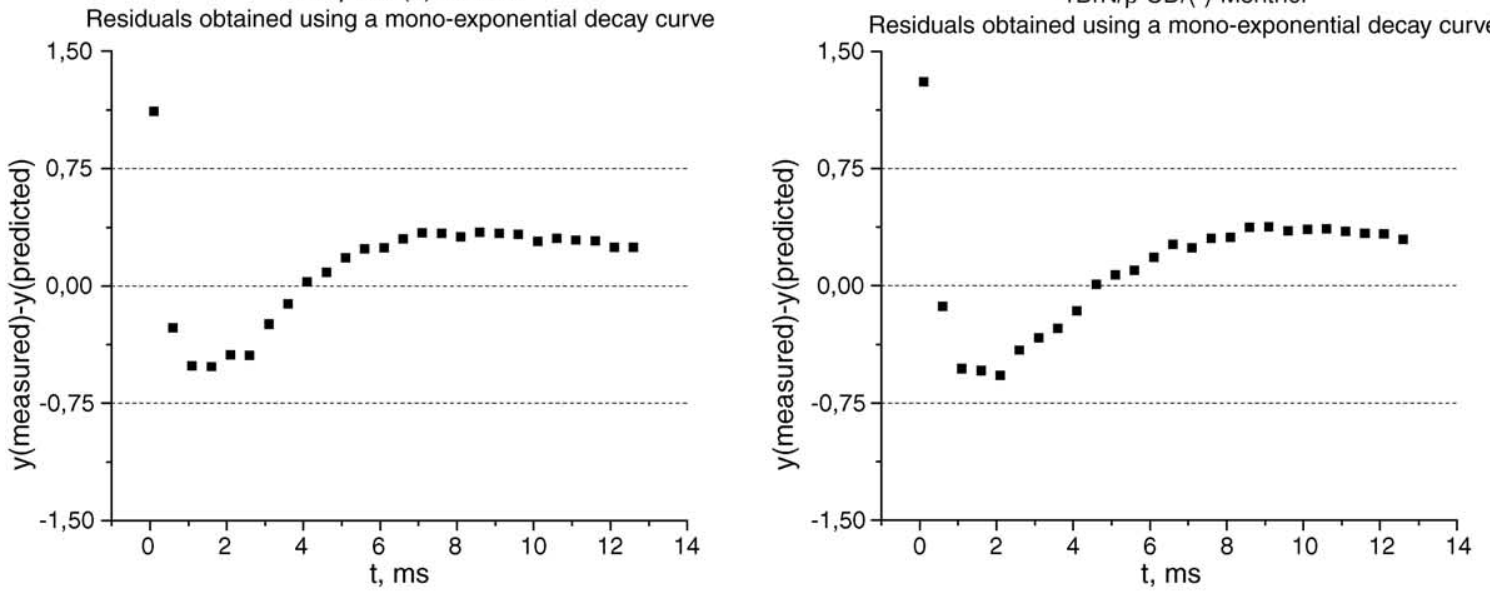

$1 \mathrm{BrN} / \mathrm{\beta}-\mathrm{CD} /(+)$-Menthol

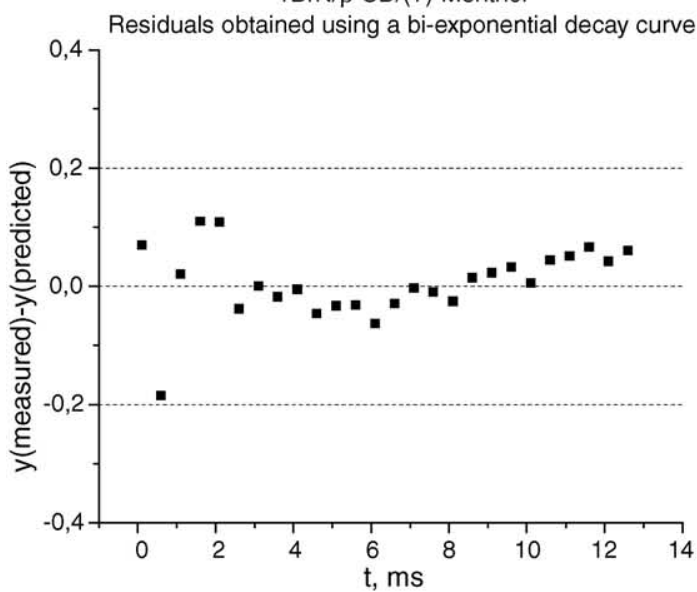

$1 \mathrm{BrN} / \mathrm{B}-\mathrm{CD} /(-)-$-Mentho

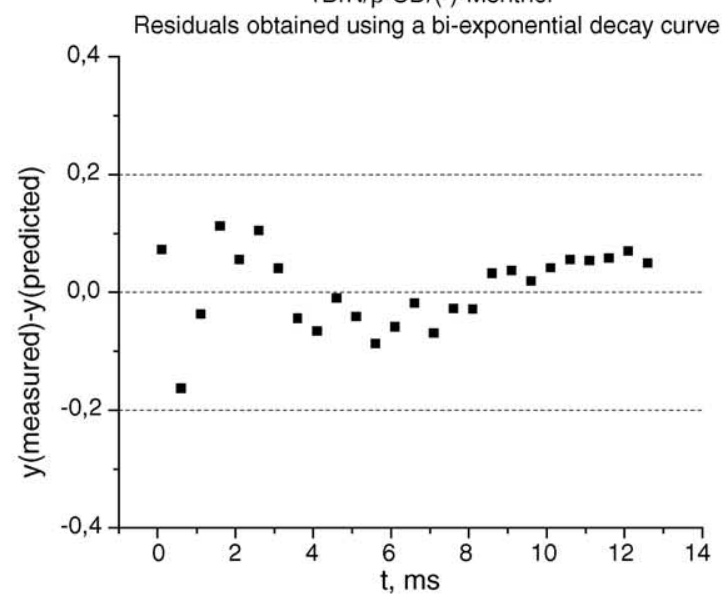

Fig. 4. Exponential decay curves and residual analysis for phosphorescence lifetimes of the $1 \mathrm{BrN} / \beta-\mathrm{CD}$ suspension in the presence of MT enantiomers under non-deoxygenated conditions. 
than that of the better protected complex with MT. Using the terminology of Table 1 and realizing that the total intensity is proportional to $\sum A_{i} \tau_{i}$, we can write:

(+)-MT (53\% increase) :

$$
1.53\left(A_{2} \tau_{2}+A_{3} \tau_{3}\right)=A_{2} \tau_{0}+A_{3} \tau_{0}
$$

(-)-MT (30\% increase) :

$$
1.30\left(A_{2} \tau_{2}+A_{3} \tau_{3}\right)=A_{2} \tau_{0}+A_{3} \tau_{0}
$$

$$
\tau=\frac{1}{\tau_{0}^{-1}+k_{\mathrm{O}_{2}}\left[\mathrm{O}_{2}\right]}
$$

in which we assume that the phosphorescence lifetime $\tau_{0}$ (in absence of quencher) will be the same for the complexes (2) and (3). Inserting the pre-exponential factors and lifetimes from Table 1 we obtain for both (+)-MT and (-)-MT a value of $4.3 \mathrm{~ms}$ for $\tau_{0}$, compared to 3.71 and $4.28 \mathrm{~ms}$ observed for these complexes under aerated conditions. It follows that in the case of the (+)-MT complex oxygen quenching plays a role in aerated suspensions: the corresponding quenching constant can be readily calculated by assuming that the oxygen concentration is about $10^{-4} \mathrm{M}$ : from Eq. (3) and a lifetime of $3.7 \mathrm{~ms}$ we obtain a quenching rate constant $k_{\mathrm{O}_{2}}$ of $4 \times 10^{5} \mathrm{M}^{-1} \mathrm{~s}^{-1}$. This value is $3-4$ orders of magnitude lower than in normal liquid solutions, but nonetheless not negligible. In the case of the (-)-MT complex, however, oxygen quenching is not significant and the observed longest lifetime is practically equal to $\tau_{0}$, apparently in this complex the phosphorophore is much better protected and no significant decrease in lifetime is observed upon aeration.

As regards possible analytical applications of the above results, it should be noted that the RTP intensity does not vary linearly with the menthol concentration. This should be expected since in the presence of oxygen mostly the fraction of MT present in the ternary complex (3) will contribute to the signal. The relative levels of the complexes (1), (2), and (3) will change with the MT concentration, resulting in an Stype curve, as depicted in Fig. 5. Apparently, while at an MT concentration of $5 \times 10^{-4} \mathrm{M}$ the concentration of the ternary complex (3) is almost at its maximum, at an MT level of $1 \times 10^{-4} \mathrm{M}$ its relative contribution is only minor because of dissociation. The two curves show a slight shift, in agreement with the (-) enantiomer forming a slightly stronger ternary complex with $1 \mathrm{BrN}$ and $\beta-\mathrm{CD}$ than the $(+)$ enantiomer.

The RTP lifetime difference under aerated conditions enables the direct determination of the MT stereochemistry. When dealing with mixtures, we have to conclude from Fig. 5 that enantiomeric ratios of (+)- and (-)-MT mixtures can only determined by RTP if the concentration of the least abundant enantiomer is at least $3 \times 10^{-4} \mathrm{M}$. At lower concentrations dissociation of the ternary complexes will hamper the distinction of the lifetimes. The range of enantiomeric ratios that will be measurable depends on the precision of the decay curve deconvolution; under the present conditions the uncertainty in

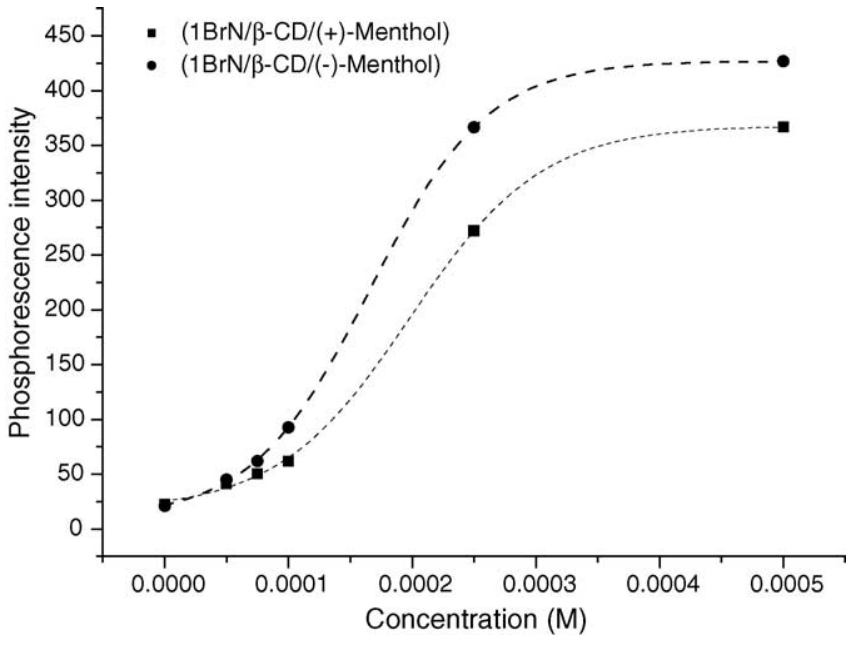

Fig. 5. Phosphorescence intensity emitted by $1 \mathrm{BrN}$ in $\beta-\mathrm{CD}$ suspension as a function of the menthol concentration. Concentrations of $\beta-\mathrm{CD}$ and $1 \mathrm{BrN}$ as usual $\left(5 \times 10^{-3}\right.$ and $5 \times 10^{-3} \mathrm{M}$, respectively).

the lifetime ( $0.06 \mathrm{~ms})$ corresponds with an enantiomeric ratio of $10 \%$. Rather unexpected, an interesting option for further improvement might be to carry out the measurements under oxygen saturated conditions instead of normal, air-saturated conditions. In that case, the oxygen concentration in the suspension will be enhanced roughly four-fold and the lifetime difference between the (+)-MT and (-)-MT complexes will become more pronounced.

\section{Conclusions}

Menthol enantiomers were found to be very effective inducers of RTP of $1 \mathrm{BrN}$ in $\beta-\mathrm{CD}$ aqueous suspension under non-deoxygenated conditions. Using this system it is possible to discriminate between the enantiomers of this nonabsorbing compound by differences in phosphorescence lifetimes of the ternary complexes. The method will probably not be suitable for trace analysis, but nevertheless it could be extremely useful for samples with higher menthol concentrations, for instance in the fragrance/food/personal care industry. The enantiomeric purity that can be handled is still limited, and in analytical practice a chiral separation step may still be necessary. The combination of such a technique with spectroscopic discrimination is well worth exploring. Similar approaches (as presently demonstrated for menthol) can be applied to discriminate between the enantiomers of other terpenes or structurally related compounds with a cyclohexane or cyclopentane ring structure.

\section{Acknowledgments}

Carmen García-Ruiz gratefully thanks the European Commission for a postdoctoral Marie Curie individual fellowship (Contract No. HPMF-CT-2002-01826). XueShan Hu thanks 
the China Scholarship Center (CSC) and NUFFIC for his grant.

\section{References}

[1] A. Ponce, P.A. Wong, J.J. Way, D.G. Nocera, J. Phys. Chem. 97 (1993) 11137.

[2] G.M. Escandar, A.M. de la Pena, Appl. Spectrosc. 55 (2001) 496.

[3] X.Z. Du, Y. Zhang, Y.B. Jiang, X.Z. Huang, G.Z. Chen, Spectrochim. Acta 53 (1997) 671.

[4] H.R. Zhang, Y.S. Wei, W.L. Jin, C.S. Liu, Anal. Chim. Acta 484 (2003) 111.

[5] G.-R. Li, J.-J. Wu, W.-J. Jin, J.-W. Xie, Talanta 60 (2003) 555.

[6] X. Du, Y. Zhan, Y. Jiang, X. Huang, G. Chen, Talanta 44 (1997) 511.
[7] G.M. Escandar, M.A. Boldrini, Talanta 53 (2001) 851.

[8] M. Santos, G.M. Escandar, Appl. Spectrosc. 55 (2001) 1483.

[9] V.B. Nazarov, V.G. Avakyan, M.V. Alfimov, T.G. Vershinnikova, Russ. Chem. Bull. 52 (2002) 916.

[10] Y.-Q. Zhai, S.-Z. Zhang, J.-W. Xie, C.-S. Liu, Anal. Chim. Acta 494 (2003) 71.

[11] W.J. Jin, J.M. Costa-Fernández, A. Sanz-Medel, Anal. Chim. Acta 431 (2001) 1.

[12] B. San Vicente de la Riva, J.M. Costa-Fernández, W.J. Jin, R. Pereiro, A. Sanz-Medel, Anal. Chim. Acta 455 (2002) 179.

[13] Fernández-Argüelles, J.M. Costa-Fernández, R. Pereiro, A. SanzMedel, Anal. Chim. Acta 491 (2003) 27.

[14] C. García-Ruiz, M.J. Scholtes, F. Ariese, C. Gooijer, Talanta 66 (2005) 641.

[15] N.J. Turro, J.D. Bolt, Y. Kuroda, I. Tabushi, Photochem. Photobiol. 35 (1982) 69. 